Veritas Et Scientia

Vol. 7, № 2, 925-934

Julio - Diciembre del 2018.

ISSN Edición Online: 2617-0639

https://doi.org/10.47796/ves.v7i2.69

\title{
DETERMINANTES DE LA SOLVENCIA FINANCIERA DE LAS CAJAS MUNICIPALES EN EL PERÚ (2012-2016)
}

DETERMINANTS OF THE FINANCIAL SOLVENCY OF THE MUNICIPAL BOXES IN PERU (2012-2016).

\author{
Paulino Celso Pinto Ranilla ${ }^{1}$ \\ Presentado: 24/03/2018 \\ Aceptado: $23 / 04 / 2018$ \\ Publicado online:27/12/2018
}

\section{RESUMEN}

El presente trabajo de investigación analiza los factores económicos y financieros que contribuyen al fortalecimiento de la solvencia financiera de las 12 cajas municipales (CMAC) que operan en el Perú y por otro lado la relación que existe entre la ratio de capital global y el puntaje $Z$ de Altman. El estudio se ha realizado con los datos de los estados financieros de los últimos cinco años (2012-2016) de cada una de las 12 instituciones que tienen vigencia al 31 de diciembre del 2016. Los resultados obtenidos, denotan que los factores que tienen mayor incidencia en el fortalecimiento de la solvencia financiera de las entidades están referidas al ingreso y a las utilidades generadas por cada una de las instituciones, así mismo, existe relación entre el puntaje $Z$ de Altman y la ratio de capital global. Se ha obtenido el modelo multifactorial que permite realizar estimaciones futuras de indicadores de solvencia financiera que acoplado al análisis de simulaciones contribuyan a la gestión de riesgos en las CMAC.

Palabras clave: Solvencia financiera, Ratio de Capital Global, Gestión de Riesgos.

\section{ABSTRACT}

The present research work analyzes the economic and financial factors that contribute to the strengthening of the financial solvency of the 12 municipal savings banks that operate in Peru and, on the other hand, the relationship

\footnotetext{
${ }^{1}$ Maestro en Dirección y Administración de Empresas
} 
that exists between the global capital ratio and the Altman $Z$ score. The study was carried out with data from the financial statements of the last five years (2012-2016) of each of the 12 institutions that are valid as of December 31, 2016. The results obtained indicate that the factors that have the greatest incidence in the strengthening of the financial solvency of the entities are related to income and profits generated by each of the institutions, likewise, there is a relationship between Altman's Z score and the global capital ratio. The multifactorial model has been obtained that allows making future estimates of financial solvency indicators that, coupled with the analysis of simulations, contribute to risk management in the CMACs.

Keywords: Financial solvency, Global Capital Ratio, Risk Management.

\section{INTRODUCCION}

A nivel internacional las diferentes versiones de BASILEA I, BASILEA II y BASILEA III se ocupan de ciertos lineamientos de gestión de riesgos financieros y del fortalecimiento de la solvencia financiera recomendado al espectro bancario. Esta preocupación ha sido más persistente en las economías después de la crisis financiera del 2008. De tal suerte, la SBS peruana ha adoptado recomendaciones y lineamientos que deben tener en cuenta los administrados a fin de minimizar sus riesgos y contribuir al fortalecimiento de la solvencia financiera de las instituciones financieras, lo que se observa a través del ratio de capital global. Desde esta perspectiva resulta siendo importante estudiar y analizar los factores que intervienen en el fortalecimiento de la solvencia financiera de las instituciones financieras.

El reporte de colocaciones a las microempresas al 15 de octubre del 2017 asciende a la suma de 2815 millones de soles (Superintendencia de Banca y Seguros (2017), Evolución del Sistema Financiero), colocaciones que ha sido dirigida al sector que significa para la economía peruana el $42 \%$ del Producto Bruto Interno, este sector comprende el $40 \%$ del sector comercial, $35 \%$ servicios y $25 \%$ manufactura y conexos. El reporte de la Superintendencia de Banca y Seguros a octubre del 2017, reporta tasas de morosidad en ascenso del sistema de cajas que van desde el $3.5 \%$ hasta más allá del $14 \%$.

Un hecho importante a resaltar es la cobertura de clientes que tienen las Cajas Municipales, que están concentradas en microempresas y éstas, tal cual hemos descrito en el párrafo anterior, han provocado a que las Cajas Municipales tengan tasas de morosidad altas, con la consecuente reducción de utilidades para las cajas. El otro hecho de singular importancia a denotar, es la competencia entre las Cajas Municipales a lo largo y ancho del país, lo que viene provocado en estas instituciones considerables disminuciones en sus utilidades y pérdida de clientes y mayores costos de gestión de créditos. Los problemas descritos en líneas antecedentes, afectó definitivamente a la solvencia financiera de las Cajas Municipales. En el Perú, de acuerdo al reporte de la SBS al 31 de octubre del 2017(Superintendencia de Banca y Seguros, Evolución del Sistema Financiero), el número de las cajas municipales vigentes con licencia de operaciones financieras son 13.

Las cajas municipales desempeñan un rol muy importante en el espectro financiero peruano. Tal es así, en el Perú mantienen operaciones activas y pasivas con las Cajas Municipales. Por otro lado, estas financieras se han constituido en el brazo financiero de las pequeñas empresas, con cuyo soporte financiero están construyendo capitales y mercados. 
La Capacidad financiera de la empresa, dada una actividad económica que genera rentabilidad positiva, se puede determinar mediante el ratio de endeudamiento y la estructura de capital de la empresa. La SBS en su tarea de supervisión valora a dos indicadores para medir la solvencia financiera, y esta son: ratio de capital global y el ratio de pasivo total/capital social y reservas. Según la SBS (2009), Resolución 4729-2009, el Ratio de Capital Global (\%), considera el patrimonio efectivo como porcentaje de los activos y contingentes ponderados por riesgo totales (riesgo de crédito, riesgo de mercado y riesgo operacional), de acuerdo con los requerimientos de Basilea II. Las instituciones financieras deben mantener un ratio de capital global mínimo de $9.5 \%$ a partir de julio de 2009 , de $9.8 \%$ a partir de julio de 2010 y desde julio de 2011, el requerimiento patrimonial exigido será de 10\%. Hasta junio de 2009 se publicaba la inversa del ratio de capital global, denominado "Apalancamiento Global", el cual no incorporaba el requerimiento de patrimonio efectivo por riesgo operacional.

"El riesgo operacional se refiere a las pérdidas potenciales resultantes de sistemas inadecuados, fallas administrativas, controles defectuosos, fraude o error humano" (Jorion, 2010).

De la revisión de la literatura respecto a la medición de la solvencia financiera de empresas, se ha revisado los estudios de (Hernández, 2014), Valencia, Venegas y Restrepo (2016), Forero (2015), Aldazábal (2014), Cruz, Pastor y Lescano(2013) y Chávez(2014) respecto al modelo de puntajes Z de Altman.

El primer marco analítico teórico se sustenta en el modelo de puntajes Z de Altman (1968 y 2000) (Elizondo, 2004), modelo que ha sido desarrollado para evaluar la solvencia financiera de las empresas. Es un modelo multifactorial. E. Altman en la Universidad de Nueva York construyó una ecuación polinómica con las variables contables para medir la probabilidad de quiebra de una empresa, que, luego tratada con la probabilidad logística, se obtiene la probabilidad de quiebra de una empresa. Un segundo marco teórico utilizado en el trabajo de investigación, es el modelo regresional de panel de datos para efectos de identificar los factores que contribuyen a la fortaleza financiera de cada una de las instituciones financieras. Este modelo de medición del impacto de ciertos factores sobre la variable solvencia financiera, busca en las empresas, las variables que inciden favorablemente y otras que no contribuyen a la solvencia financiera.

Gitman(2012), en el modelo de Panel Data, incluye los ratios de Rentabilidad de patrimonio, Relación entre la utilidad neta respecto al patrimonio de la empresa y Ratio de rentabilidad del activo. Se cnoce que los factores que afectan al sistema financiero son diversas, tal como la volatilidad de los mercados financieros, las variables de la política monetaria, que es el caso del comportamiento de la tasa de interés, las variables de la inflación, la evolución del crecimiento de una economía, la institucionalidad, así como la oferta y la demanda del dinero. (López, 1997, p.22).

\section{OBJETIVOS}

a) Determinar por cada institución financiera los puntajes de Altman y su relación con el nivel de solvencia financiera histórica mostrada por las Cajas Municipales en el Perú en el periodo 2012-2016.

b) Identificar en las Cajas Municipales los factores económicos y financieros que contribuyen a la fortaleza financiera de las Cajas Municipales en el periodo 2012-2016.

\section{METODOLOGÍA}


linvestigación descriptiva, explicativa, cuantitativa y diseño no experimental. El ámbito de estudio comprende las Cajas Municipales que vienen operando activamente, según reporte de la SBS al cierre del 31 de diciembre del 2016, que incluye a 11 cajas municipales y la Caja Metropolitana de Lima. Se trabajó con un tamaño de la muestra por conveniencia de 12 Cajas Municipales operativas al cierre del año 2016. Recojo sistemático y cronológico de los estados financieros de las instituciones financieras en estudio. Para la aplicación del modelo Z de Altman, se recogieron los datos de los estados financieros reportados a la SBS por las cajas municipales dentro del periodo 2012 a 2016, información financiera anual, de los estados de situación financieros y estado de resultados. Para la aplicación del modelo de Panel Data, se utilizan las ratios de rendimiento obtenidos a partir de los estados financieros anualizados. Se obtiene los estados financieros anuales de las cajas municipales del repositorio de la SBS de los periodos comprendidos en 2012 hasta 2016, para lo que se tiene en cuenta el modelo Z1 de

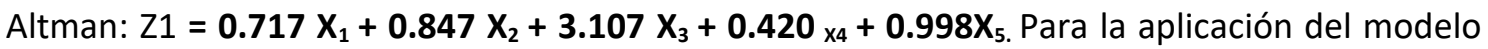
de Panel Data, se utilizó el modelo: $Z_{i t}=\beta_{i t}+\beta_{i t} X_{i t}+\ldots+\beta_{i t} X_{i t}+€_{i t}$

\section{RESULTADOS}

\section{RATIO DE CAPITAL GLOBAL DE LAS CAJAS MUNICIPALES.}

La estructura financiera de toda institución financiera está constituida por una alta proporción del pasivo y por una proporción pequeña de capital. La mayoría de las CMAC mantiene el capital a niveles del $14 \%$, ciertamente por encima del valor mínimo del $10 \%$ exigido por la SBS y comparativamente están en los niveles de la banca corporativa. La proporción del pasivo se mantiene en el rango de $80 \%$ a $90 \%$, lo que quiere decir, que hay instituciones que han preferido operar con $20 \%$ de patrimonio y $80 \%$ de deuda, por otro lado, también hay entidades que han mostrado preferencias por operar a niveles de exigencia mínima del $10 \%$ de capital exigido por la SBS (Fig. 01).

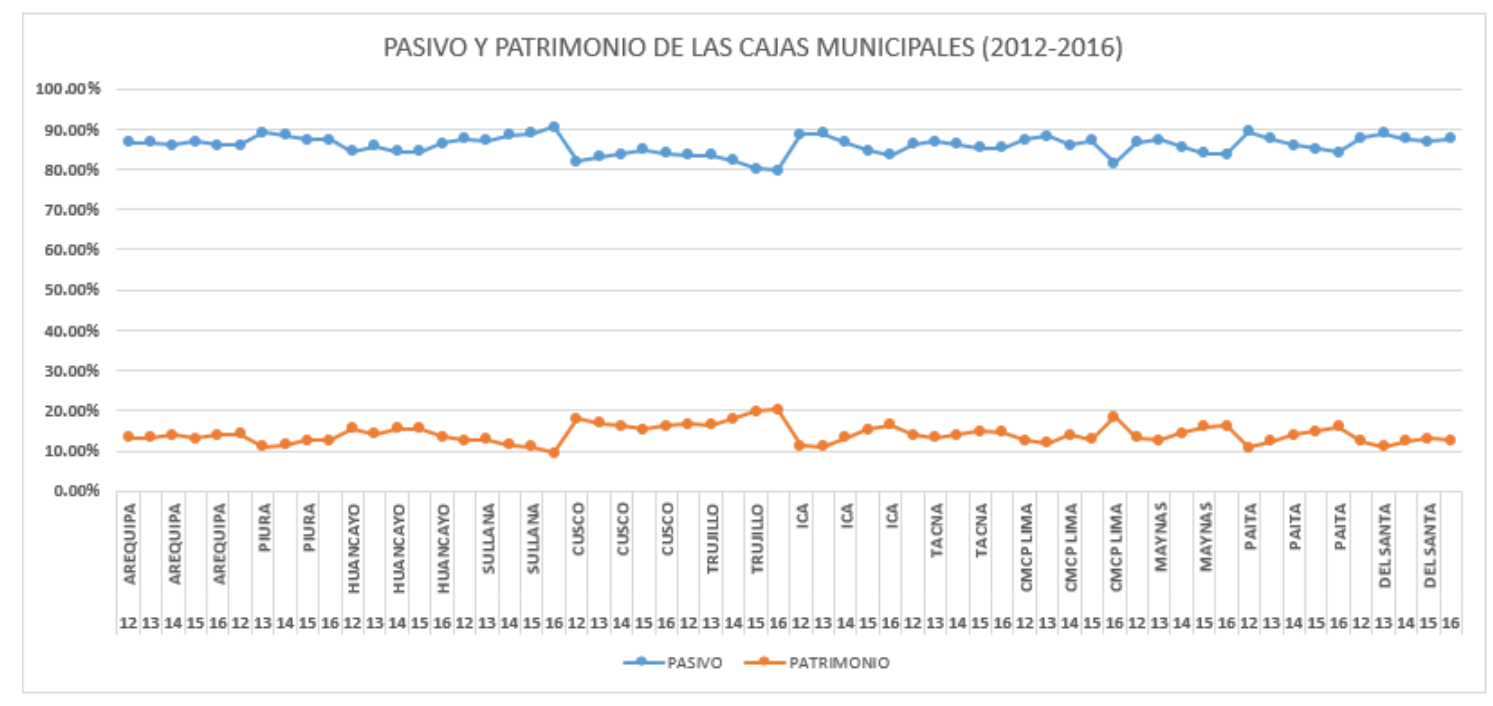

\section{Figura 1}

Estructura financiera de las CMAC 2012-2016

Se puede apreciar a la CMAC Arequipa, en el periodo analizado, manteniendo el pasivo en el orden de $86 \%$ y patrimonio de $14 \%$, que es el rango que exhibe la Banca Corporativa en el Perú. Por otro lado, a partir de la misma figura arriba presentada, se observa a la CMAC Sullana 
exhibiendo niveles de pasivo en $90 \%$ y patrimonio a $10 \%$, siendo el mínimo exigido por la SBS. El caso más notorio es la de CMAC Trujillo que mantiene el rango del pasivo alrededor de $80 \%$ y patrimonio del orden de $20 \%$, el doble del exigido por la SBS. (Fig. 02)

Se puede apreciar de manera notoria, la evolución del pasivo de la CMAC Paita que ha venido disminuyendo hasta llegar a $14 \%$ en el año 2016 , en otras palabras, ha venido fortaleciendo su capital a través de los cinco últimos años. La mayoría de las CMACs de esta segunda mitad exhiben patrimonio en el nivel de $15 \%, 5 \%$ por encima del requerimiento mínimo exigido por la SBS y muy cercano a los niveles de la banca corporativa. (Fig. 03)

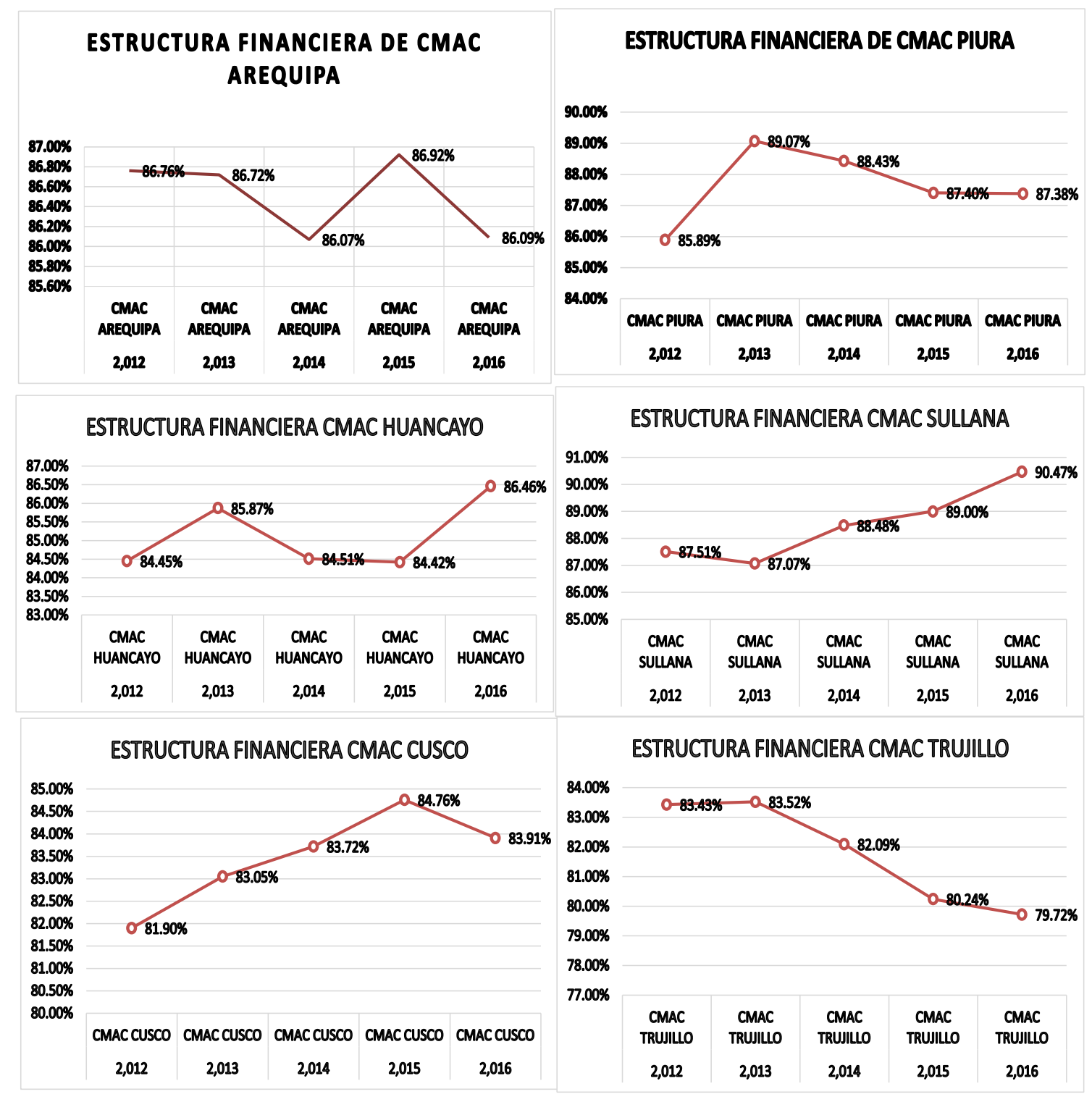

Figura 2

Estructura financiera de las primeras 6 CMACs del Ranking 


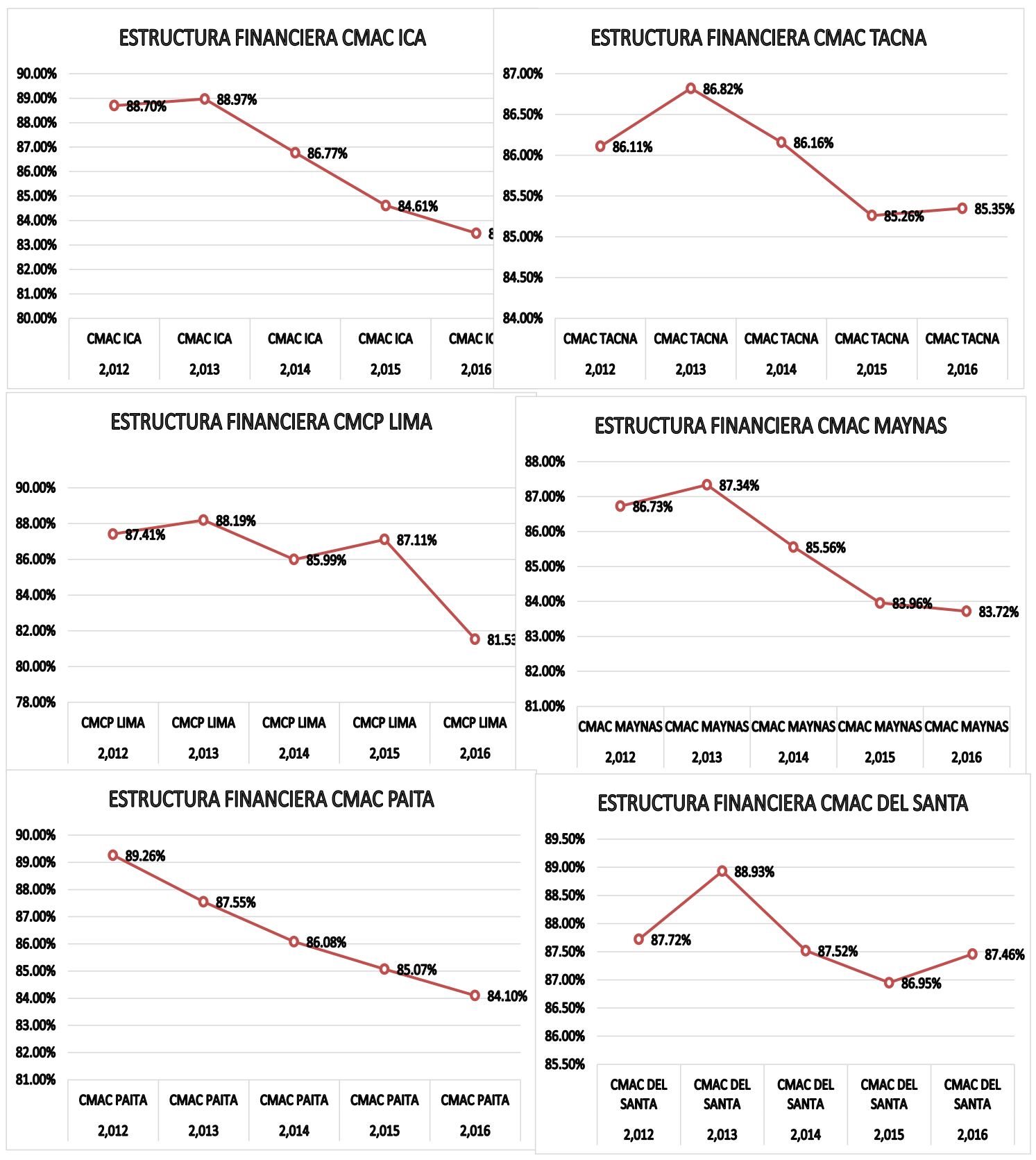

Figura 3

Estructura financiera de la segunda mitad de las CMACs del Ranking.

El Ratio de Capital Global del periodo 2012 fue de alrededor del 18\% corresponde a la CMAC Trujillo y CMAC Cusco y el más bajo a la CMAC Paita con valor de 10.41\%. En el 2013 la institución que tiene el más alto valor es la CMAC Trujillo con $17.72 \%$ y el más valor exhibido le corresponde a la CMAC Paita con 11.06\%. En el 2014 observamos que la CMAC Trujillo es la que tiene el máximo valor de $17.41 \%$ y el más bajo valor le corresponde a la CMAC Sullana con 11.79\%. En el 2015 el valor más alto del ratio de capital global corresponde a la CMAC Trujillo con valor de $19.22 \%$ y el valor más bajo a CMAC Sullana con $12.42 \%$. En el 2016 la CMAC Trujillo exhibe el valor más alto de $19.80 \%$ y el valor más bajo le corresponde a la CMAC Del Santa con $13.13 \%$. 
Tabla 1

Resultados de puntaje Z1 de Altman (2012-2016)

\begin{tabular}{|r|l|c|c|c|c|c|}
\hline \multicolumn{1}{|l|}{ AÑO } & INSTITUCION & \multicolumn{1}{|c|}{ Z1 } & \multicolumn{1}{c|}{$\mathbf{X 1}$} & \multicolumn{1}{c|}{ X2 } & \multicolumn{1}{c|}{ X3 } & \multicolumn{1}{c|}{ X5 } \\
\hline 2012 & CMAC AREQUIPA & 1.16882 & 0.95533 & 0.02710 & 0.11247 & 0.11166 \\
\hline 2013 & CMAC AREQUIPA & 1.25135 & 0.94476 & 0.02687 & 0.11546 & 0.19286 \\
\hline 2014 & CMAC AREQUIPA & 1.02347 & 0.94464 & 0.02208 & 0.04136 & 0.19934 \\
\hline 2015 & CMAC AREQUIPA & 0.92697 & 0.85885 & 0.02408 & 0.03964 & 0.16794 \\
\hline 2016 & CMAC AREQUIPA & 1.02583 & 0.94555 & 0.02557 & 0.04673 & 0.18137 \\
\hline 2012 & CMAC PIURA & 1.17824 & 0.92124 & 0.01633 & 0.12342 & 0.12065 \\
\hline 2013 & CMAC PIURA & 1.22130 & 0.92844 & 0.01548 & 0.11674 & 0.18014 \\
\hline 2014 & CMAC PIURA & 0.91330 & 0.93128 & 0.01697 & 0.01697 & 0.17882 \\
\hline 2015 & CMAC PIURA & 1.01340 & 0.93321 & 0.01947 & 0.04678 & 0.18283 \\
\hline 2016 & CMAC PIURA & 1.25038 & 0.93220 & 0.01885 & 0.12654 & 0.17320 \\
\hline 2012 & CMAC HUANCAYO & 1.25223 & 0.95246 & 0.03141 & 0.13331 & 0.12877 \\
\hline 2013 & CMAC HUANCAYO & 1.23353 & 0.96193 & 0.02786 & 0.11429 & 0.16545 \\
\hline 2014 & CMAC HUANCAYO & 1.30687 & 0.96091 & 0.03240 & 0.13128 & 0.18294 \\
\hline 2015 & CMAC HUANCAYO & 1.33399 & 0.95935 & 0.03627 & 0.14011 & 0.18046 \\
\hline 2016 & CMAC HUANCAYO & 1.01817 & 0.96301 & 0.02716 & 0.04405 & 0.16818 \\
\hline 2012 & CMAC SULLANA & 1.12397 & 0.94203 & 0.01850 & 0.10607 & 0.10351 \\
\hline 2013 & CMAC SULLANA & 1.22213 & 0.93981 & 0.01387 & 0.11049 & 0.19364 \\
\hline 2014 & CMAC SULLANA & 1.19617 & 0.94105 & 0.0171516 & 0.10804 & 0.17157 \\
\hline 2015 & CMAC SULLANA & 1.24115 & 0.94349 & 0.01884 & 0.11764 & 0.18356 \\
\hline 2016 & CMAC SULLANA & 1.17833 & 0.94699 & 0.01583 & 0.10484 & 0.16049 \\
\hline
\end{tabular}

Se puede apreciar que, en este primer grupo del primer tercio, el mejor puntaje de Z1 de Altman le corresponde a CAMC Huancayo a lo largo de los 5 años analizados. El más bajo puntaje le corresponde a CMAC Piura en el año 2015. (Tabla 01)

Tabla 2

Puntaje Z1 de Altman (el segundo tercio de las CMAC).

\begin{tabular}{|c|c|c|c|c|c|}
\hline \begin{tabular}{|l|l|}
2012 & CMAC CUSCO \\
\end{tabular} & 1.13142 & 0.94715 & 0.01256 & 0.10759 & 0.10759 \\
\hline 2013 CMAC CUSCO & 1.20285 & 0.94924 & 0.02948 & 0.10914 & 0.15849 \\
\hline 2014 CMAC CUSCO & 1.20678 & 0.95031 & 0.02941 & 0.10992 & 0.15928 \\
\hline 2015 CMAC CUSCO & 0.99408 & 0.95165 & 0.02817 & 0.04098 & 0.16090 \\
\hline 2016 CMAC CUSCO & 1.02845 & 0.98554 & 0.02802 & 0.04186 & 0.16836 \\
\hline \begin{tabular}{|l|l|}
2012 & CMAC TRUJILLO \\
\end{tabular} & 1.16523 & 0.94783 & 0.02624 & 0.11450 & 0.10788 \\
\hline \begin{tabular}{l|l|}
2013 & CMAC TRUJILLO \\
\end{tabular} & 1.19827 & 0.94787 & 0.01792 & 0.10587 & 0.17489 \\
\hline 2014 CMAC TRUJILLO & 1.21976 & 0.94214 & 0.01726 & 0.11362 & 0.17698 \\
\hline 2015 CMAC TRUJILLO & 1.24807 & 0.93980 & 0.02009 & 0.12027 & 0.18392 \\
\hline \begin{tabular}{|l|l|}
2016 & CMAC TRUJILLO \\
\end{tabular} & 1.24720 & 0.93174 & 0.02192 & 0.12591 & 0.16970 \\
\hline 2012 CMAC ICA & 1.05188 & 0.93986 & 0.01156 & 0.09090 & 0.08596 \\
\hline 2013 CMAC ICA & 1.14659 & 0.93903 & 0.01019 & 0.09095 & 0.18245 \\
\hline 2014 CMAC ICA & 1.01191 & 0.93520 & 0.01616 & 0.04364 & 0.19249 \\
\hline 2015 CMAC ICA & 1.04060 & 0.93483 & 0.02336 & 0.04976 & 0.19634 \\
\hline 2016 CMAC ICA & 1.02685 & 0.92287 & 0.02061 & 0.05070 & 0.19054 \\
\hline 2012 CMAC TACNA & 1.09389 & 0.93873 & 0.02280 & 0.09810 & 0.09692 \\
\hline 2013 CMAC TACNA & 1.12033 & 0.93090 & 0.01122 & 0.08762 & 0.17147 \\
\hline 2014 CMAC TACNA & 1.13517 & 0.91403 & 0.01249 & 0.09159 & 0.18503 \\
\hline 2015 CMAC TACNA & 0.83571 & 0.90249 & -0.00118 & 0.00185 & 0.18423 \\
\hline 2016 CMAC TACNA & 0.89066 & 0.90826 & 0.00904 & 0.02029 & 0.16908 \\
\hline
\end{tabular}

De este segundo grupo de los valores Z1 de Altman, que el más alto puntaje le corresponde a CMAC Trujillo con valor promedio de 1.24, el puntaje más bajo le corresponde a CMAC Tacna 
con promedio 1.0. Todas las CMAC se encuentran en la zona roja, a excepción de CAMC Trujillo que se encuentra en la zona gris. (Tabla 2)

Tabla 3

Puntaje Z1 de Altman Tercer grupo CMAC 2012-2016

\begin{tabular}{|r|l|r|r|r|r|r|}
\hline 2012 & CMCP LIMA & 0.95334 & 0.86889 & 0.00205 & 0.08164 & 0.07512 \\
\hline 2013 & CMCP LIMA & 0.82835 & 0.93090 & 0.00014 & 0.00014 & 0.16068 \\
\hline 2014 & CMCP LIMA & 1.12251 & 0.87427 & -0.01462 & 0.10474 & 0.18297 \\
\hline 2015 & CMCP LIMA & 1.16702 & 0.83920 & -0.01616 & 0.12125 & 0.20268 \\
\hline 2016 & CMCP LIMA & 1.22562 & 0.81773 & -0.02995 & 0.14456 & 0.21594 \\
\hline 2012 & CMAC MAYNAS & 1.19180 & 0.93544 & 0.01770 & 0.12448 & 0.11958 \\
\hline 2013 & CMAC MAYNAS & 1.19007 & 0.93986 & 0.00870 & 0.10276 & 0.18992 \\
\hline 2014 & CMAC MAYNAS & 1.26983 & 0.92690 & 0.01204 & 0.12804 & 0.19762 \\
\hline 2015 & CMAC MAYNAS & 1.03521 & 0.92786 & 0.01675 & 0.05073 & 0.19851 \\
\hline 2016 & CMAC MAYNAS & 0.96718 & 0.92078 & 0.00777 & 0.03424 & 0.19439 \\
\hline 2012 & CMAC PAITA & 1.00383 & 0.88627 & -0.00950 & 0.09312 & 0.08726 \\
\hline 2013 & CMAC PAITA & 1.17217 & 0.89644 & -0.00153 & 0.10365 & 0.20910 \\
\hline 2014 & CMAC PAITA & 1.24027 & 0.86422 & 0.00034 & 0.13108 & 0.21350 \\
\hline 2015 & CMAC PAITA & 0.92258 & 0.87001 & -0.00487 & 0.02688 & 0.21984 \\
\hline 2016 & CMAC PAITA & 0.91298 & 0.87066 & -0.00516 & 0.02551 & 0.21425 \\
\hline 2012 & CMAC DEL SANTA & 1.06754 & 0.91242 & 0.00127 & 0.10189 & 0.09587 \\
\hline 2013 & CMAC DEL SANTA & 1.14132 & 0.93057 & -0.00607 & 0.09475 & 0.18521 \\
\hline 2014 & CMAC DEL SANTA & 1.30730 & 0.91267 & 0.00893 & 0.14497 & 0.19531 \\
\hline 2015 & CMAC DEL SANTA & 1.24630 & 0.89958 & 0.00684 & 0.12691 & 0.20161 \\
\hline 2016 & CMAC DEL SANTA & 1.18222 & 0.89033 & -0.02513 & 0.11325 & 0.21371 \\
\hline
\end{tabular}

En el tercer grupo de evaluación de puntajes Z1 de Altman, se puede apreciar valores máximos del puntaje le corresponde a la CMAC Del Santa en los cinco años analizados, el valor más bajo de los puntajes en los cinco años le corresponde a la CMAC Paita. Todas las CMAC de este tercer grupo están en la zona roja, excepto la CMAC Del Santa.

Según la relación que existe entre el ratio de capital global y el puntaje Z1 de Altman, a lo largo de los cinco años analizados para las doce cajas municipales, la evolución histórica guarda una similar tendencia, del tipo de series de tiempo no estacionarias. (Fig. 04)

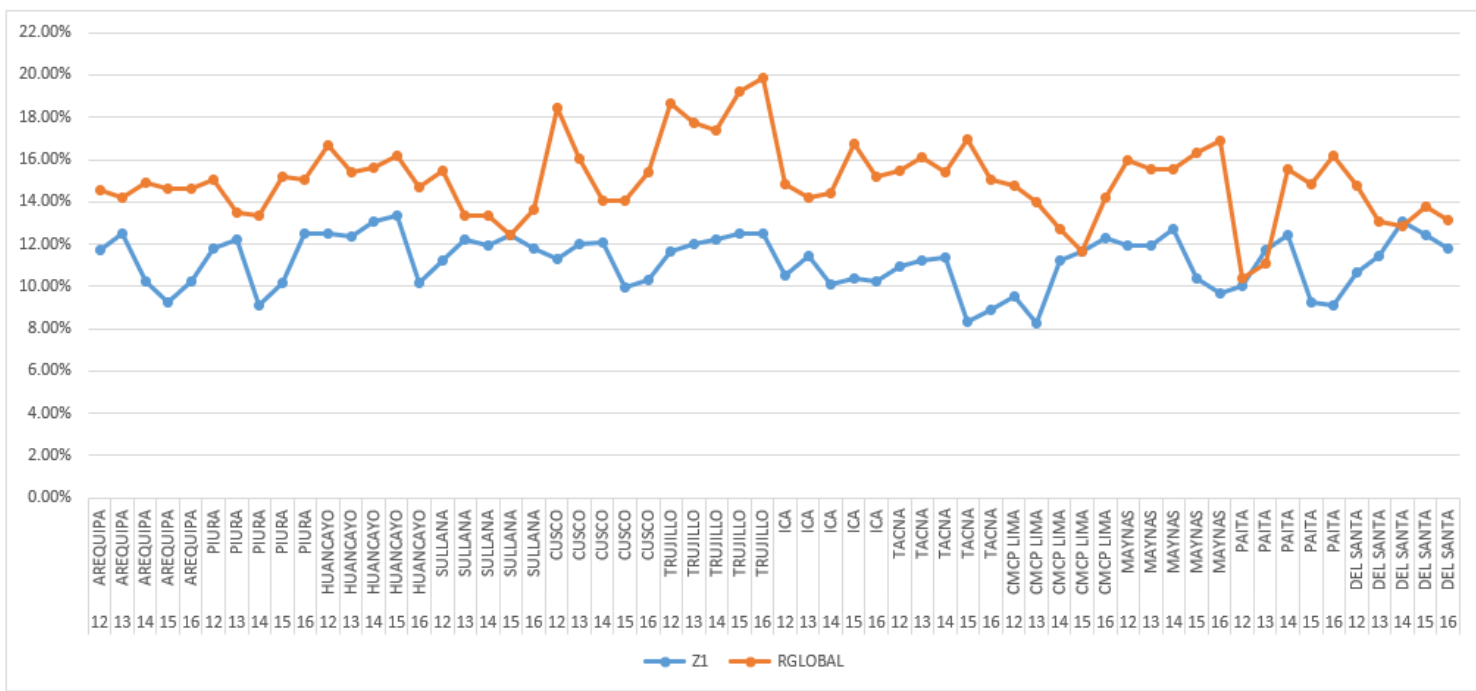

Figura 4

Ratio de Capital Global y Puntaje Z1 de Altman. 
Los resultados de la regresión en panel de datos, nos ilustra claramente que la fortaleza financiera está influenciada negativamente por el ratio de solvencia financiera y positivamente por la zona de Altman en el que se ubican. (Tabla 4)

\section{Tabla 4}

\section{Relación de solvencia financiera y la fortaleza financiera de Altman}

\begin{tabular}{|c|c|c|c|c|}
\hline \multicolumn{5}{|c|}{$\begin{array}{l}\text { Dependent Variable: } Z 1 \\
\text { Method: Panel Least Squares } \\
\text { Date: } 12 / 08 / 17 \text { Time: 08:17 } \\
\text { Sample: } 20122016 \\
\text { Periods included: } 5 \\
\text { Cross-sections included: } 12 \\
\text { Total panel (balanced) observations: } 60\end{array}$} \\
\hline Variable & Coefficient & Std. Error & t-Statistic & Prob. \\
\hline $\begin{array}{c}\text { C } \\
\text { RGLOBAL } \\
\text { ZONA }\end{array}$ & $\begin{array}{r}1.111169 \\
-0.012985 \\
0.154978\end{array}$ & $\begin{array}{l}0.163445 \\
0.010938 \\
0.026923\end{array}$ & $\begin{array}{r}6.798445 \\
-1.187179 \\
5.756261\end{array}$ & $\begin{array}{l}0.0000 \\
0.2418 \\
0.0000\end{array}$ \\
\hline \multicolumn{5}{|c|}{ Effects Specification } \\
\hline \multicolumn{5}{|c|}{$\begin{array}{l}\text { Cross-section fixed (dummy variables) } \\
\text { Period fixed (dummy variables) }\end{array}$} \\
\hline $\begin{array}{l}\text { R-squared } \\
\text { Adjusted R-squared } \\
\text { S.E. of regression } \\
\text { Sum squared resid } \\
\text { Log likelihood } \\
\text { F-statistic } \\
\text { Prob(F-statistic) }\end{array}$ & $\begin{array}{l}0.657942 \\
0.519491 \\
0.087090 \\
0.318558 \\
72.01257 \\
4.752137 \\
0.000020\end{array}$ & \multicolumn{2}{|c|}{$\begin{array}{l}\text { Mean dependent var } \\
\text { S.D. dependent var } \\
\text { Akaike info criterion } \\
\text { Schwarz criterion } \\
\text { Hannan-Quinn criter. } \\
\text { Durbin-Watson stat }\end{array}$} & $\begin{array}{r}1.120379 \\
0.125637 \\
-1.800419 \\
-1.172115 \\
-1.554655 \\
1.823924\end{array}$ \\
\hline
\end{tabular}

\section{DISCUSIÓN}

A partir del modelo obtenido, se observa que la fortaleza financiera de las instituciones financieras en gran medida depende principalmente de la capacidad de generar utilidad utilizando el activo y en segundo término de la capacidad de generar ingresos utilizando el activo. Del análisis de los antecedentes, tanto Forero (2015) y Hernández (2014), el modelo Z de Altman han permitido realizar el análisis de solvencia financiera de las empresas a fin de que los gestores de las empresas puedan tener en cuenta para la toma de decisiones orientadas al fortalecimiento financiero de las empresas.

Las variables que tienen mayor importancia y aquellas que tienen una menor importancia para la solvencia financiera de las instituciones financieras, destacan la variable que denota la capacidad de generar utilidad por parte del activo y en segundo lugar de importancia la capacidad de generar ingresos por parte del activo. En el periodo estudiado, la CMAC Huancayo es la única institución que ha evolucionado dentro de la zona gris hacia la zona verde, es decir, es la institución con mayor solvencia financiera de entre las 12 cajas estudiadas. Así mismo se constata que la CMAC Tacna es la que viene estando en la zona roja en los cinco periodos estudiados. Las demás CMAC se encuentran en el tiempo a veces en zona gris y en otras en zona roja, que es concordante con el ratio de capital global exigido por la 
SBS. Se tiene claro la importancia de generar utilidad e ingresos por parte de las instituciones financieras, para el fortalecimiento de la solvencia financiera de las instituciones financieras. Se sugiere utilizar el modelo $\mathrm{Z1}$, porque tiene una alta correlación dinámica con el requerimiento de capital global de la SBS y Basilea II y III. Se sugiere tener en cuenta que el fortalecimiento financiero de las instituciones financieras está altamente explicado por la utilidad y por los ingresos.

\section{REFERENCIAS BIBLIOGRÁFICAS}

Chávez, V. (2014). Reforzamiento Patrimonial para las Cajas Municipales de Ahorro y Crédito, PUCP. Perú.

Cruz, V., Pastor, R. y Lescano, V. (2013). Estimación de Solvencia Financiera para Evaluar el Riesgo de Quiebra de Empresas peruanas, tesis para optar el grado de magister en Finanzas Corporativas en la UPC.

Elizondo, A. (2004). Medición Integral del Riesgo de Crédito. México: editorial Limusa.

Forero, J. (2015). Evaluación de Insolvencia Financiera de MYPES. Colombia.

Gitman, L. (2013). Principios de Administración Financiera. México: editorial Pearson.

Gujarati, D. (2012). Econometría: México: editorial McGraw Hill.

Hernández, H. (2014). Modelo Financiero para la Detección de Quiebras con el uso de Análisis Discriminante Múltiple. Costa Rica.

Jorion, P. (2010). Valor en Riesgo. México: editorial Limusa.

Ministerio de la Producción, Perú (2013). Las Mipymes en Cifras.

SBS (2017). Evolución del Sistema Financiero. Perú.

SBS (2012-2016). Ratio de Capital Global del Sistema Financiero Peruano. Perú

SBS (2009). Resolución No. 4729-2009, El Ratio de Capital Global. Perú

SBS (2009). Resolución No. 2115. Perú

SBS (2009). Resolución No. 14354. Requerimiento de Capital Global. Perú

SBS (2006). Basilea II hacia Basilea III. Perú.

SMV (1992). DL. No. 26126. Ley de Mercado de Valores del Perú. Perú.

Valencia, M., Venegas, J. y Restrepo., J. (2016). Solvencia Financiera de Pequeñas Empresas Agroindustriales, Colombia: Antioquía.

Venegas, F. (2006). Riesgos Financieros y Económicos. México: editorial Thomson. 